\title{
Direct activation of STING in the tumor microenvironment partially overcomes immune tolerance in neu-N transgenic mice
}

\author{
Jeremy Foote ${ }^{1 *}$, James M Leatherman² ${ }^{2}$ Todd D Amstrong ${ }^{2}$, Laureen Ojalvo ${ }^{3}$, David Kanne ${ }^{4}$, Elizabeth M Jaffee ${ }^{5}$, \\ Tom Dubensky ${ }^{4}$, Leisha Emens ${ }^{3}$
}

From 30th Annual Meeting and Associated Programs of the Society for Immunotherapy of Cancer (SITC 2015) National Harbor, MD, USA. 4-8 November 2015

STING signaling induces interferon- $\beta$ production by intratumoral dendritic cells (DCs), driving $\mathrm{T}$ cell priming within the tumor microenvironment. However, the impact of antigen-specific tolerance on this process is not well studied. We therefore examined the efficacy of in situ tumor delivery of a potent STING-activating CDN ligand (ML-RR-S2 CDA) in both non-tolerant parental $\mathrm{FVB} / \mathrm{N}$ and the immune tolerant neu- $\mathrm{N}$ transgenic mice bearing established HER-2 $2^{+}$breast tumors. In nontolerant $\mathrm{FVB} / \mathrm{N}$ mice, single agent intratumoral ML-RRS2 CDA injection induced complete tumor regression of both the injected tumor and a contralateral uninjected tumor, while also protecting mice from a subsequent tumor challenge. In contrast, intratumoral injection of ML-RR-S2 CDA alone in neu-N mice modestly delayed tumor growth. Furthermore, in contrast to our prior data that low dose cyclophosphamide could increase vaccine-induced immunity and tumor-free survival in neu- $\mathrm{N}$ transgenic mice, the sequential delivery of low dose cyclophosphamide with ML-RR-S2 CDA did not delay tumor outgrowth relative to ML-RR-S2 CDA alone. We therefore explored biomarkers of both STING pathway activation and $\mathrm{T}$ cell activity within the TME of FVB/N and neu-N transgenic mice. Intratumoral ML-RR-S2 CDA injection resulted in both high levels of IFN $\beta$ production in the TME of tumor bearing $\mathrm{FVB} / \mathrm{N}$ mice, and the induction of a durable population of HER-2-specific $C D 8^{+} \mathrm{T}$ cells. In contrast, intratumoral ML-RR-S2 CDA injection resulted in low levels of IFNb production in the TME of tolerant neu-N transgenic mice, and the induction of few HER-2-specific
$\mathrm{CD}^{+} \mathrm{T}$ cells. Phenotypic analyses of tumor infiltrating leukocytes within the TME of untreated neu-N mice revealed increased expression of $\mathrm{PD}-1$ on $\mathrm{CD}^{+} \mathrm{T}$ cells and PD-L1 on myeloid cells. Moreover, about $25 \%$ of HER $-2^{+}$tumor cells derived from the TME also expressed PD-L1. The addition of PD-L1 blockade to low dose cyclophosphamide in sequence with intratumoral ML-RR-S2 CDA resulted in a greater delay of tumor growth than intratumoral ML-RR-S2 CDA injection alone in neu- $\mathrm{N}$ transgenic mice, but did not clear any mouse of tumor. These findings suggest that multiple mechanisms of immune tolerance limit the ability of STING pathway activation to lead to HER-2 specific$\mathrm{CD}^{+} \mathrm{T}$ cell activation and tumor regression in tolerant neu- $\mathrm{N}$ transgenic mice.

\section{Authors' details}

'Department of Molecular and Comparative Pathobiology, Johns Hopkins School of Medicine, Baltimore, MD, USA. ${ }^{2}$ Johns Hopkins University, Baltimore, MD, USA. ${ }^{3}$ Department of Oncology, Johns Hopkins School of Medicine, Baltimore, MD, USA. ${ }^{4}$ Aduro Bio Tech, Berkley, CA, USA. ${ }^{5}$ The Sidney Kimmel Comprehensive Cancer Center at Johns Hopkins, Baltimore, MD, USA.

Published: 4 November 2015

doi:10.1186/2051-1426-3-S2-P356

Cite this article as: Foote et al.: Direct activation of STING in the tumor microenvironment partially overcomes immune tolerance in neu-N transgenic mice. Journal for ImmunoTherapy of Cancer 2015 3(Suppl 2): P356. 